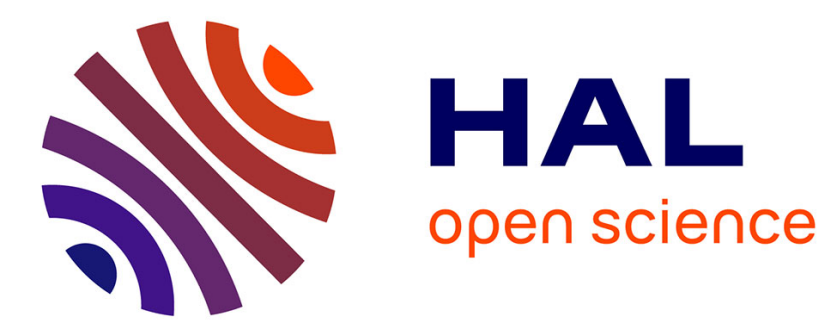

\title{
An Automatic Multilevel Refinement Technique based on Nested Local Meshes for Nonlinear Mechanics
}

Laureline Barbié, Isabelle Ramière, Frédéric Lebon

\section{To cite this version:}

Laureline Barbié, Isabelle Ramière, Frédéric Lebon. An Automatic Multilevel Refinement Technique based on Nested Local Meshes for Nonlinear Mechanics. Computers \& Structures, 2015, 147, pp.14-25. 10.1016/j.compstruc.2014.10.008 . hal-01247907

\section{HAL Id: hal-01247907 \\ https://hal.science/hal-01247907}

Submitted on 30 May 2018

HAL is a multi-disciplinary open access archive for the deposit and dissemination of scientific research documents, whether they are published or not. The documents may come from teaching and research institutions in France or abroad, or from public or private research centers.
L'archive ouverte pluridisciplinaire $\mathbf{H A L}$, est destinée au dépôt et à la diffusion de documents scientifiques de niveau recherche, publiés ou non, émanant des établissements d'enseignement et de recherche français ou étrangers, des laboratoires publics ou privés. 


\title{
An automatic multilevel refinement technique based on nested local meshes for nonlinear mechanics
}

\author{
L. Barbié ${ }^{\mathrm{a}, \mathrm{b}}$, I. Ramière ${ }^{\mathrm{a}, *}$, F. Lebon ${ }^{\mathrm{b}}$ \\ ${ }^{a}$ CEA, DEN, DEC, SESC, F-13108 Saint-Paul Lez Durance, France \\ ${ }^{\mathrm{b}}$ LMA, CNRS, UPR 7051, Aix-Marseille Univ, Centrale Marseille, 31, Chemin Joseph Aiguier, F-13402 Marseille Cedex 20, France
}

\begin{abstract}
In this paper an adaptive multilevel mesh refinement method, coupled with the Zienkiewicz and Zhu a posteriori error estimator, is applied to solid mechanics with the objective of conduct reliable nonlinear studies in acceptable computational times and memory space. Our automatic approach is first verified on linear behaviour, on 2D and 3D simulations. Then a nonlinear material behaviour is studied. Advantages and limitations of the local defect correction method in solid mechanics problems in terms of refinement ratio, error level, CPU time and memory space are discussed. This kind of resolution is also compared to a global h-adaptive resolution.
\end{abstract}

\section{Introduction}

Adaptive refinement methods (e.g. [1-5]) are devoted to solve problems with various characteristics length-scale in acceptable computational times and memory space. The aim of this paper is to deal with problems having local discontinuities. Among the refinement methods available, those inducing small elements (h-refinement method [1,6] for example) are more efficient to simulate local discontinuities than those increasing the order of the polynomial basis (p-refinement method [2,7] for example). Moreover the pollution error [8,9] (i.e. the residual error due to the non-refined part of the problem) depends on the mesh size and may be controlled by refining the element size but not by modifying the order of the polynomial basis. That is the reason why we decided to use adaptive mesh refinement (AMR) techniques. One of the constraints of the study was to use an existing industrial solver, that means to change only pre-processing and post-processing operations. Thus, we chose to use local multi-grid methods [4] consisting in generating local sub-grids with finer and finer discretisation step. Furthermore, it induces simple meshes (uniform, structured and regular). As we study elliptic problems discretised by the finite element (FE) method, it is interesting to use structured regular meshes for the reason that it produces well-defined problems. The local defect correction (LDC) $[10,4]$

\footnotetext{
* Corresponding author.
}

method was retained because it is not specific to flux conservative problems.

The refinement process is piloted thanks to the well-known Zienkiewicz and Zhu (ZZ) a posteriori error estimator [11] founded on stress smoothing.

This paper is based upon Barbié et al. [12] but the current version includes results obtained from additional research on the efficiency of the coupled LDC-ZZ method for linear behaviour (see also [13]), a further study on three-dimensional test case where different initial meshes are compared and an extended work concerning the nonlinear test case, particularly with the use of refinement ratio greater than 2 .

\section{Adaptive mesh refinement approach}

\subsection{Local defect correction method}

The local defect correction (LDC) method was introduced by Hackbusch [10]. Its convergence was also proved by Hackbusch [14]. It is based on the multi-grid process [15]. A global coarse grid is used on the whole domain, and only local fine sub-grids are set on areas where more precision is required. An example of nested grids is shown on Fig. 1. The local fine grid lies on a zone of interest defined on the coarse grid. Such type of local sub-grid can be defined recursively until reaching the desired accuracy. 
Prolongation and restriction operators are defined to link several levels of computation. Coarse and fine problems are then sequentially computed until the solution converges on the coarser grid. Such an iterative process is traditionally represented by a $\wedge$-cycle, as on Fig. 2.

\subsubsection{Problem definition}

Let us consider the problem $(\mathcal{P})$ defined on an open domain $\Omega$ of boundary $\Gamma$ :

$$
(\mathcal{P}):\left\{\begin{array}{l}
\mathcal{L}(u)=f \text { in } \Omega \\
\text { B.C. on } \Gamma
\end{array}\right.
$$

with:

$$
\begin{cases}\mathcal{L} & : \text { usually nonlinear operator } \\ u & : \text { solution } \\ f & : \text { right-hand member } \\ \text { B.C. } & : \text { boundary conditions }\end{cases}
$$

A set of nested domains $\Omega_{l}, 0 \leqslant l \leqslant l^{*}$, with $\Omega_{0}=\Omega$ and $l^{*}$ the number of levels, is then defined. Each domain is discretised by a grid $G_{l}$ of boundary $\Gamma_{l}$. The space step $h_{l}$ of the mesh $G_{l}$ is defined by $h_{l}=h_{l-1} / r=h_{0} / r^{l}$ with $r$ the refinement ratio. The local discrete problem on each grid $G_{l}$ at iteration $k$ writes:

$\left(\mathcal{P}_{l}^{k}\right):\left\{\begin{array}{l}\mathcal{L}_{l}\left(u_{l}^{k}\right)=f_{l}^{k} \text { in } G_{l} \\ \text { appropriate B.C. on } \Gamma_{l}\end{array}\right.$

where $\mathcal{L}_{l}$ is the discrete operator associated to $\mathcal{L}_{\mid \Omega_{l}}$ on $G_{l}$ and $f_{l}^{0}=f_{\mid G_{l}}$.

The boundary conditions will be specified during the prolongation step while the right-hand member $f_{l}^{k}$ will be defined during the restriction step.

\subsubsection{Prolongation step: boundary conditions}

As recommended by [13], at the prolongation step the problem $\left(\mathcal{P}_{l}^{k}\right)$ is solved with $f_{l}^{k}=f_{l}^{k-1}$.

On the coarsest grid $G_{0}$, the boundary conditions of the whole problem are applied.

The boundary conditions on the other grids $G_{l}, 1 \leqslant l \leqslant l^{*}$ are represented on Fig. 3:

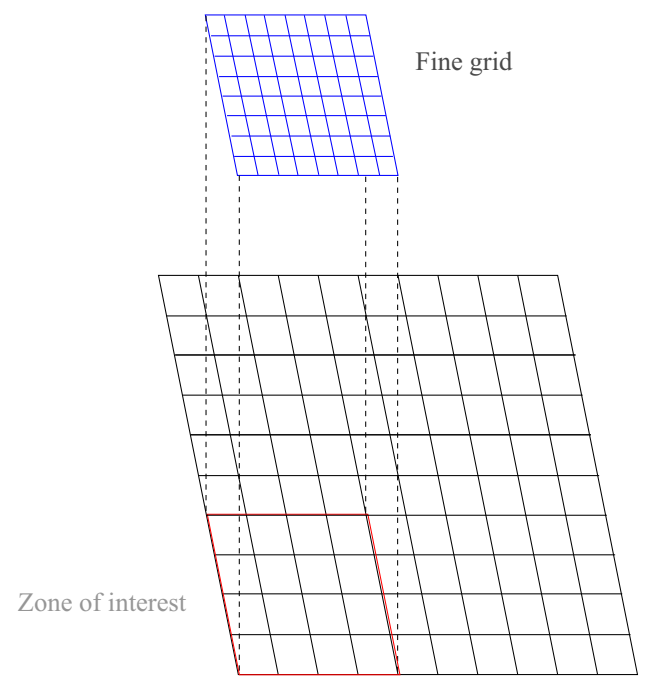

Coarse grid

Fig. 1. Example of nested meshes used in LDC method.

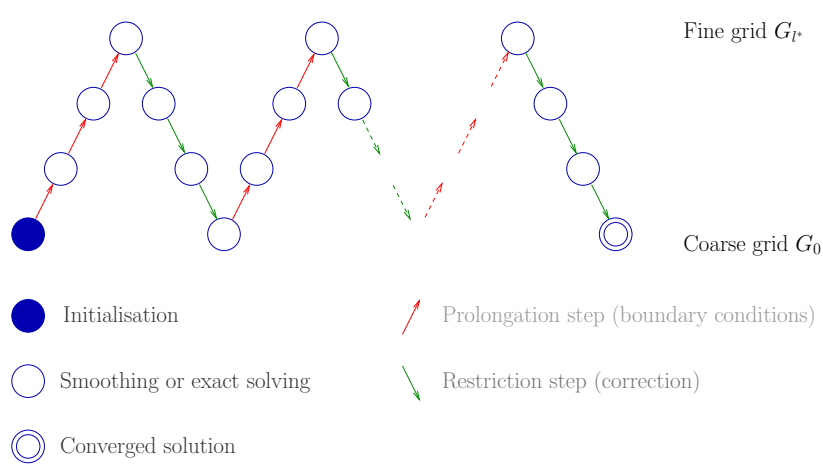

Fig. 2. Representation of LDC process: $\wedge$-cycles.

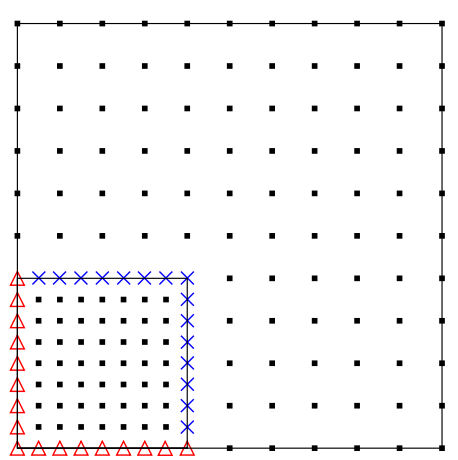

$\triangle$ Continuous problem boundary conditions

$\times$ Projection of coarse problem solution

Fig. 3. Prolongation step: boundary conditions on $G_{l}(l \neq 0)$

- If $\Gamma_{l} \cap \Gamma \neq \varnothing$, the boundary conditions of the original problem $(\mathcal{P})$ are used.

- On the other part of the boundary, Dirichlet boundary conditions are applied. A projection operator $P_{l-1}^{l}$ applied on the next coarser solution $u_{l-1}^{k}$ enables us to obtain the Dirichlet values.

\subsubsection{Restriction step: defect correction}

At the restriction step, the boundary conditions defined on the prolongation step are kept to solve the problem $\left(\mathcal{P}_{l}^{k}\right)$. For each grid level $l, 0 \leqslant l \leqslant l^{*}-1$, the restriction step consists in correcting the right-hand side of the problem $\left(\mathcal{P}_{l}^{k}\right)$ via a defect calculated from the next finer solution $u_{l+1}^{k}$.

Two sets of nodes of $G_{l}$ have to be defined, see Fig. 4. $A_{l}$ contains the nodes of the grid $G_{l}$ strictly included on the domain discretised by $G_{l+1}$. $\AA_{l}$ is made up of the interior nodes of $A_{l}$ (in the sense of the discretisation scheme).

First, the solution of the problem $\left(\mathcal{P}_{l+1}^{k}\right)$ is restricted to the nodes of $A_{l}$ :

$\tilde{u}_{l}^{k}(x)=\left(R_{l+1}^{l} u_{l+1}^{k}\right)(x) \quad \forall x \in A_{l}$
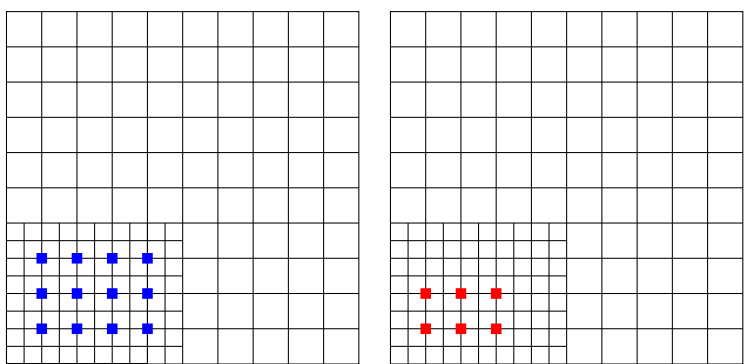

Fig. 4. Restriction zone $A_{l}$ on the left and correction zone $A_{l}^{\circ}$ on the right (e.g. for operator $\Delta$ ). 
where $R_{l+1}^{l}$ is a restriction operator on the solution from the grid $G_{l+1}$ to the grid $G_{l}$.

The local defect associated to this restriction is then computed on the nodes of $A_{l}^{\circ}$. As the fine problem may be only smoothed, the defect $\hat{r}_{l+1}^{k}$ of the fine problem also impacts the correction term:

$$
\begin{aligned}
r_{l}^{k}(u)(x)= & \underbrace{\left(\mathcal{L}_{l}\left(\tilde{u}_{l}^{k}\right)-f_{l}^{0}\right)(x)}_{\text {coarse defect associated to fine solution }} \\
& -\underbrace{\left(\hat{R}_{l+1}^{l} \hat{r}_{l+1}^{k}\right)(x)}_{\text {projection of fine defect }} \forall x \in A_{l}^{\circ}
\end{aligned}
$$

with:

$\hat{r}_{l+1}^{k}=\mathcal{L}_{l+1}\left(u_{l+1}^{k}\right)-f_{l+1}^{k}$

and $\hat{R}_{l+1}^{l}\left(\neq R_{l+1}^{l}\right)$ a restriction operator from the fine grid $G_{l+1}$ to the coarse grid $G_{l}$ dedicated to source term interpolation. This correction term is similar to the one used in the standard multi-grid Full Approximation Scheme [15], and the condition $\hat{R}_{l+1}^{l}=\left(P_{l}^{l+1}\right)^{T}$ has to be respected in order to obtain the convergence.

Remark: If the problem can be easily solved exactly or quasiexactly on each grid (elastic linear behaviour for example), the fine defect $\hat{r}_{l+1}^{k}$ becomes then negligible [4].

Then the coarse solution $u_{l}^{k}$ is obtained by solving the coarse problem $\left(\mathcal{P}_{l}^{k}\right)$ with the modified right-hand member:

$f_{l}^{k}=f_{l}^{0}+\chi_{A_{l}} r_{l}^{k}(u)$

where $\chi_{A_{i}}$ is the characteristic function of $A_{l}^{\circ}: \chi_{A_{l}}(x)=\left\{\begin{array}{ll}1 & \text { if } x \in A_{l}^{\circ} \\ 0 & \text { elsewhere }\end{array}\right.$.

As the correction term only applies on $A_{l}^{\circ}$, this formulation can not be simplified into a correction equation even for linear problems.

Prolongation and restriction operators are usually interpolation operators, for which the degree of interpolation has to be in agreement with the expected order of the discretisation method [16].

\subsection{Zienkiewicz and Zhu a posteriori error estimator}

In order to detect automatically the zone of interest which will defined the local sub-grids, an a posteriori error estimator is used. This kind of estimator is devoted to estimate a measurement of the discretisation error. The Zienkiewicz and Zhu (ZZ) $[11,17,18]$ a posteriori error estimator was selected because it is not time consuming and it is easy to plug in a black-box context, compared to the other existing error estimators (e.g. $[19,20])$.

The $\mathrm{ZZ}$ a posteriori error estimator consists in constructing a stress solution $\sigma^{*}$ more regular than the FE one $\sigma_{h}$. The local estimator $\eta_{E}$ on an element $E$ is defined as:

$\eta_{E}=\left\|\sigma_{h}^{*}-\sigma_{h}\right\|_{L^{2}(E)}$

The element value is obtained from nodes values. For more details, the reader is referred to $[11,17,18]$.

\section{Test case}

\subsection{Physical considerations}

The pellet-cladding interaction ( $\mathrm{PCI}$ ) [21] appears during irradiation in pressurised water reactors, which are the essential of french nuclear reactors. The fuel is formed of cylindrical pellets of $8.2 \mathrm{~mm}$ diameter, composed of uranium dioxide $\left(\mathrm{UO}_{2}\right)$, piled up in a zircaloy cladding. Two phenomena lead to $\mathrm{PCI}$ :
- The fuel pellet cracks and swells as soon as the irradiation begins (see on Fig. 7 left). The external pressure imposed by the water induces the cladding creeping, which results in a discontinuous contact between the pellet and the cladding.

- Another phenomenon, illustrated by Fig. 5 adds discontinuities. As the fuel pellet has a finite axial size, the temperature gradient leads to a hourglass shape deformation of the pellet. Thus, the contact between the fuel and the pellet appears first in front of the inter-pellet plane. The hourglass shape phenomenon results in a concentration of stresses around the inter-pellet plane.

The PCI can produce the cladding failure. As the cladding is the first confinement barrier of the irradiated fuel, modelling precisely the PCI is of great importance. Research and development on this subject are then still undertaken worldwide. From a numerical point of view, complete 3D simulations are currently limited because of the required unstructured and irregular meshes, inducing ill-conditioned systems with an important number of degrees of freedom. The LDC method seems then well suited for this kind of application.

\subsection{Numerical and algorithmic considerations}

In all this study, a simplified PCI model is used. We are only interested in obtaining precise simulations of the cladding response to the pellet modifications. The elastic part of the cladding behaviour is ruled by the Young's modulus $E=100 \mathrm{GPa}$ and the Poisson's ratio $v=0.3$. The effect of the pellet is represented a discontinuous pressure on the internal radius of the cladding.

The finite element solver used for this study is CAST3M [22], the mechanical software developed by the CEA (French Atomic Energy and Alternative Energies Commission). Quadrangular bilinear $Q_{1}$ finite elements are used for modelling reasons [23]. In order to be more realistic compared to industrial simulations where the pellet position is a priori unknown, the meshes used are non-fitted to pressure discontinuities. The location of the pressure discontinuity will be approximated by the mesh: the distance $d_{h}$ on a mesh of size step $h$ represents the distance between the real location and the mesh approximated location of the pressure jump. Hence, two meshes with different mesh steps $h$ and $h^{\prime}$ may have $d_{h}=d_{h^{\prime}}$ as for the example presented on Fig. 6.

Concerning the LDC algorithm, we have chosen to work on hierarchical meshes between each level of refinement in order to simplify the restriction and prolongation steps. As the mechanical

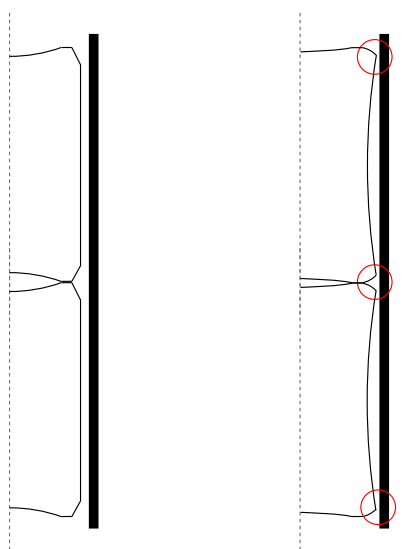

Fig. 5. Illustration of the hourglass shape phenomenon: before (left) and after (right) irradiation. 


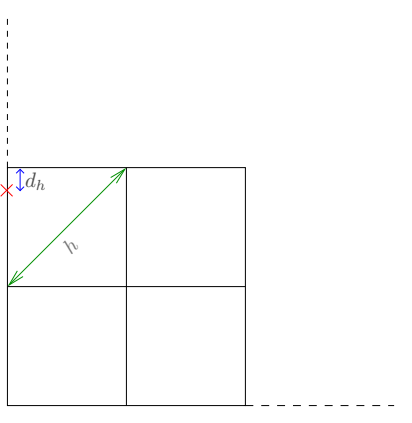

$\times$ Pressure discontinuity

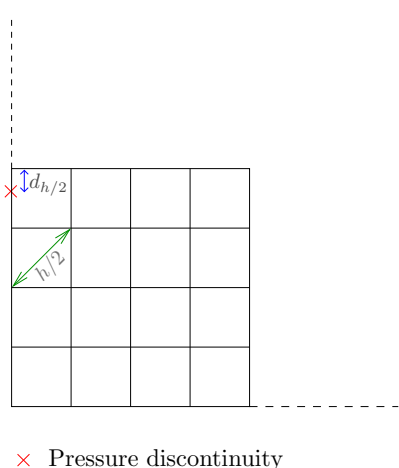

$\times$ Pressure discontinuity
Fig. 6. Definition of the distance $d_{h}$ to the pressure jump defined by the mesh of size $h$. In this example $d_{h}=d_{h / 2}$.

problem is formulated in terms of displacement, the solution $u$ of problem (1) represents here the displacement vector.

Each local problems is solved exactly (limited number of degrees of freedom). Hence, the prolongation and restriction operators only concern the displacements: no conservation principle has to be respected. A bilinear interpolation based on the polynomial finite element basis functions is used for the prolongation operator $P_{l-1}^{l}$ while a canonical injection is used for the restriction operator $R_{l+1}^{l}$. These operators are in good agreement with the expected first-order accuracy of the method, see [24].

As, to the best of our knowledge, the LDC method has never been applied to solid mechanics (except in [13]), part 4 is devoted to verify the efficiency and the convergence of the method for a linear elastic behaviour of the cladding. These results are complementary in more practical sense than those described in [13]. Two-dimensional and three-dimensional test cases will be under study. Then, the LDC method will be extended to nonlinear behaviour in Section 5 .

\section{Verification study}

In this paper, we focus on the effects on the cladding of the pellet cracking phenomenon and of the complete three-dimensional pellet modifications (cracking and hourglass shape phenomenons). For more details about the own effects on the cladding of the pellet hourglass configuration, the reader is referred to $[12,13]$.

In all this section, the behaviour of the cladding is supposed to be linear elastic. No volume force is applied.

\subsection{Bi-dimensional analysis}

\subsubsection{Problem definition}

This model is under the plane strain hypothesis in the $2 D(r, \theta)$ plane. As the geometry of the cladding is curved in this plane (see Fig. 7), the meshes used are regular structured but only "quasi" uniform. The goal is to verify the LDC method on a non academic case, particularly when the geometry has to be approximated. Indeed, one of the main drawbacks of hierarchical meshes is that the curvature approximation will not be improved during the sub-grids generation.

The boundary conditions with the cracked pellet is represented by a pressure discontinuity on the internal radius of the cladding, in front of the crack opening. As the pellet is assumed to crack in a regular way, see [25], only $1 / 16$ of the cladding is represented $(0 \leqslant \theta \leqslant \pi / 8)$. On Fig. 7 , the geometry of the problem and the boundary conditions are available. These boundary conditions can be summarized as follows with $\mathbf{u}$ the displacement vector, $\sigma$ the Cauchy stress tensor, $\mathbf{n}$ the outward normal unit vector, $R_{\text {int }}=4.1 \mathrm{~mm}, e=0.6 \mathrm{~mm}, R_{\text {ext }}=R_{\text {int }}+e=4.7 \mathrm{~mm}, f=8 \mu \mathrm{m}$ and $\theta_{f}=\arctan \left(\frac{f}{R_{\text {int }}}\right)$ :

- Symmetry conditions due to the partial representation of the cladding:

$\mathbf{u} \cdot \mathbf{n}=\mathbf{0}$ on $\theta=0$ and $\theta=\pi / 8, \forall r$

- Internal discontinuous pressure representing the contact with the cracked pellet:

$\sigma \mathbf{n}=0$ Pa on $r=R_{\text {int }} \quad$ and $\quad 0 \leqslant \theta \leqslant \theta_{f}$

$\sigma \mathbf{n}=-80 \cdot 10^{6}$ Pa on $r=R_{\text {int }}$ and $\theta_{f}<\theta \leqslant \pi / 8$

- External pressure imposed by the coolant:

$\sigma \mathbf{n}=-15.5 \cdot 10^{6}$ Pa on $r=R_{\text {ext }} \quad \forall \theta, 0 \leqslant \theta \leqslant \pi / 8$

\subsubsection{Mesh convergence study}

On Fig. 8, an example of nested meshes used for our simulations can be seen. At each level, the current mesh is in black and the zone of interest is in green. This refinement zone is obtained selecting the elements $L \subset G_{l}$ that respect:

$\eta_{L}>\alpha\left(\max _{K \subset G_{l}} \eta_{K}-\min _{K \subset G_{l}} \eta_{K}\right)$

where $\eta_{L}$ is the local ZZ error in stress defined in (7). Thanks to the sensibility study made by Barbié et al. [13], we set $\alpha=0.25$. In order

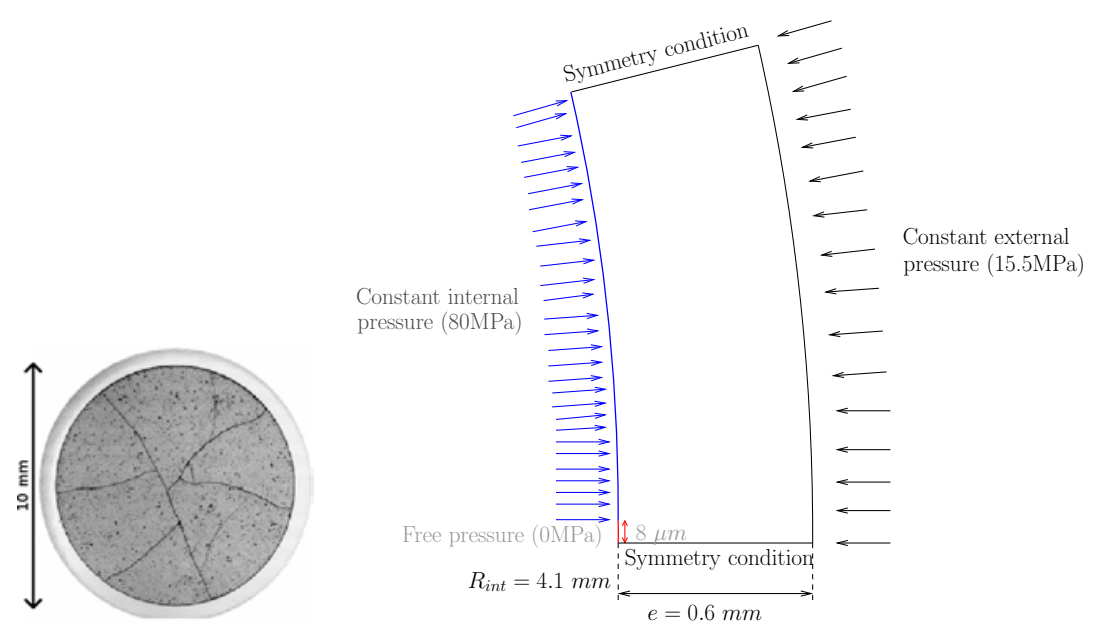

Fig. 7. Problem definition - 2D $(r, \theta)$ plane strain model. 

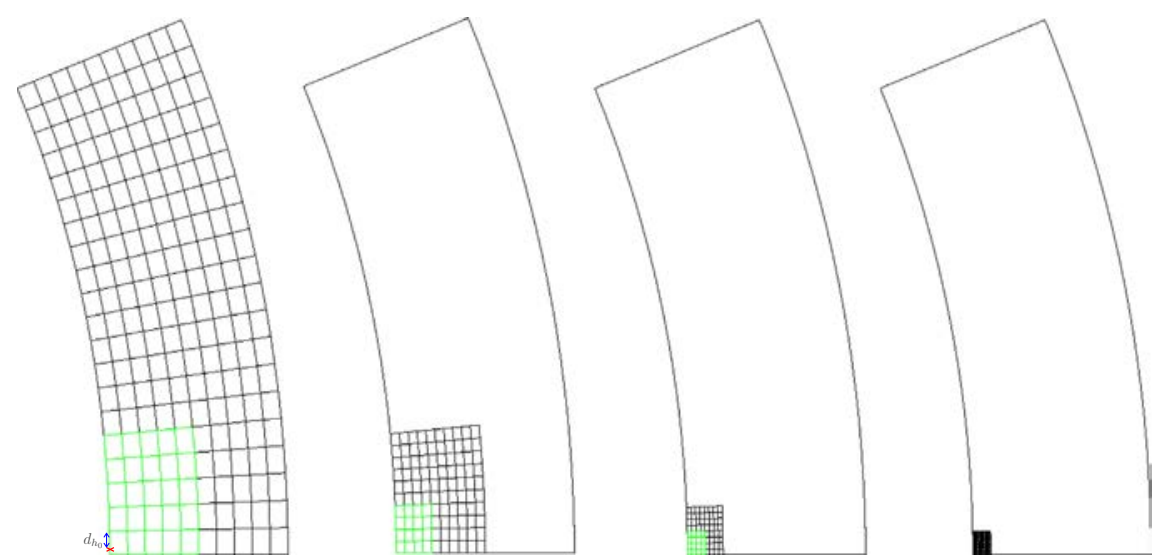

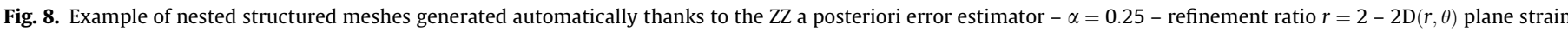
model.

to obtain nested structured meshes, some elements have to be added to the selected ones. Indeed, working with structured regular grids allows to avoid numerical artifacts due to reflex corners and to increase the speed up of the solver. We can notice here that refinement zones are very localised. Moreover, if the refinement zones are compared to a crack observed experimentally in a cladding after irradiation (see Fig. 9), we can observe that these zones are in good coherence with the stress concentration area in the cladding. This gives confidence in a further analysis of failure initiation.

In this case, to verify our approach, we aim to compute errors on the obtained LDC solutions in displacement. We could use the analytical solution developed by Roberts [26]. However, this solution is written with a Fourier decomposition, and we cannot perform the required number of terms to obtain an enough precise solution. So, we consider as reference solution the $Q_{1}$ FE solution obtained on a fine mesh of cell size $1 \mu \mathrm{m}$ in each direction $(\simeq 2$ millions of degrees of freedom (DoF)), adapted to the size of the pressure discontinuity $(8 \mu \mathrm{m})$.

In [13], it had been shown that for this kind of discretisation scheme, the $L^{2}$ and $L^{\infty}$ norms are equivalent. In the sequel, we only present results in $L^{\infty}$ norm which is meaning from an engineering viewpoint because it guarantees a maximal local error. The relative maximal error between this reference displacement and the composite LDC displacement, noted $\left\|e_{h}\right\|_{L^{\infty} \text {.comp }}$, is plotted on Fig. 10 according to the distance $d_{h_{0}}$ of the coarse mesh to be refined (see Section 3.2 and Figs. 6 or 8 ) and for a refinement ratio $r=2$. In the sequel, $h_{i}$ will denote a mesh step of $218 \mu \mathrm{m}$. From each of the five initial coarse meshes $\left(l^{\sqrt{*}}=0\right)$, one to four local sub-grids have been generated.

The first conclusion to be drawn is that the method converges at the first-order with respect to the coarse mesh distance $d_{h_{0}}$ to the discontinuity. The loss of one order of convergence compared to the standard $Q_{1}$ FE resolution was expected since the location of the pressure jump is approximated by the mesh, as mentioned by Ramière [24].

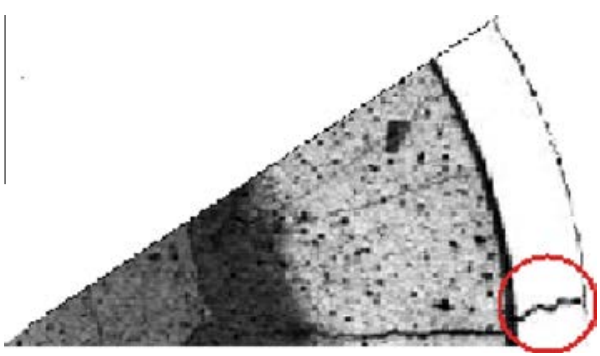

Fig. 9. Example of crack observed on a cladding after irradiation.

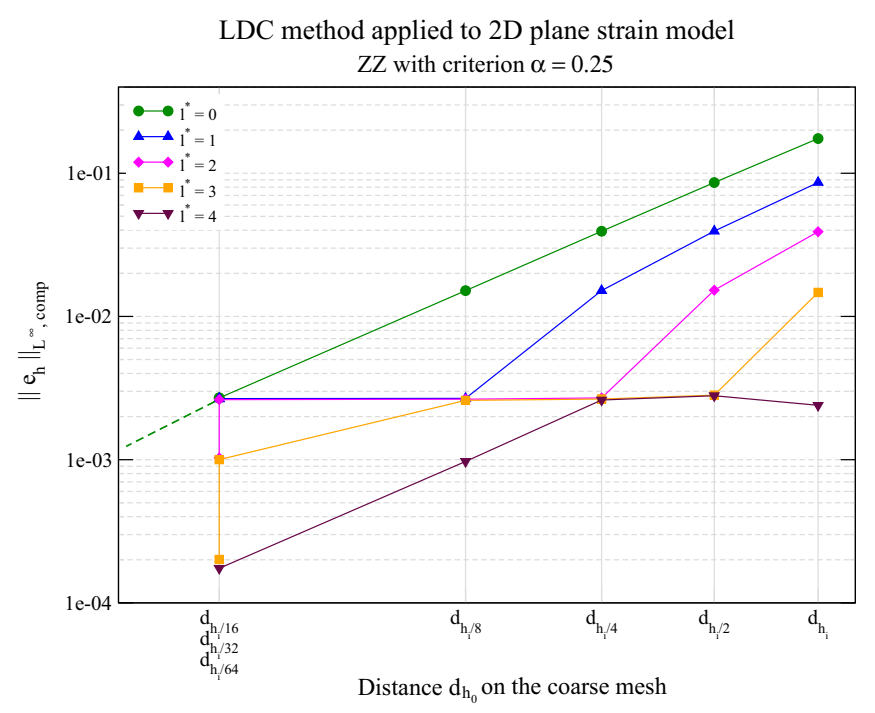

Fig. 10. $L^{\infty}$ error on the composite grid according to the distance to the discontinuity on the coarsest mesh - 2D plane strain model.

The second conclusion is that the LDC method conserves the order of convergence with respect to the local finest distance to the discontinuity. Indeed the same error level is obtained with a local refinement as with a global one with a discretisation step equal to the local finest one, as observed in [27]. Thus, the LDC method converges as $O\left(d_{h_{\text {fine }}}\right)$, where $d_{h_{\text {fine }}}$ corresponds to the distance to the discontinuity on the local finest mesh. This conclusion remains true even for an important number of sub-grids or an important decrease of the error.

The observed stagnation is due to the fact that the error is controlled by the local distance to the discontinuity. Then, even if the local mesh step is divided, if the distance to the discontinuity remains unchanged, the error will not decrease.

Finally, even for small relative error $\left(<1 \cdot 10^{-3}\right)$, the error due to the coarse approximation of the geometry remains negligible compared to the error due to jump position approximation. That confirms the choice of using hierarchical meshes.

\subsubsection{Interest of sub-grids}

To optimise the ratio precision obtained over CPU time, the Fig. 11 represents the composite $L^{\infty}$ error with respect to CPU time and to the number of sub-grids. 
From this figure, we can conclude that the more the expected precision is restrictive, the more the use of an initial coarse mesh with many sub-grids is advantageous.

As the memory space is also an important constraint in the simulation process, we then study the number of nodes required by each LDC simulation. The Table 1 represents the total number of nodes (sum of all the grids) and the additional nodes for each new sub-grid.

This table confirms that the same behaviour is obtained for the memory space than for the time consuming. For example, to get the same precision than with a quasi uniform mesh of size $h_{i} / 16$, we need 22 times less nodes using an initial mesh of size $h_{i} / 2$ and 3 sub-grids, or 12 times less with an initial mesh of size $h_{i} / 4$ and $l^{*}=2$ or 4 times less with an initial mesh of size $h_{i} / 8$ and $l^{*}=1$. We also gain $80 \%$ of the maximal number of nodes per grid using an initial mesh of size $h_{i} / 2$ and 3 sub-grids instead of a quasi uniform mesh of size $h_{i} / 16$. We can notice that the first row of the table has a slightly different behaviour. The coarsest initial mesh $\left(h_{0}=h_{i}\right)$ is so rough that it needs first to be totally refined, which induces more nodes than with an initial mesh of size $h_{i} / 2$, whatever the number of sub-grids.

Moreover, we can verify that the sub-grids are very localised (100 to 200 nodes for most of these sub-grids). It confirms that to accurately simulate in a cheapest way problems with local discontinuities, a progressive mesh refinement is really efficient.

\subsubsection{Comparison with a global h-adaptive refinement technique}

According to the previous conclusions made on Fig. 11 and Table 1, it seems more attractive to use a coarse initial mesh and a lot of sub-grids. Moreover, the use of refinement ratio greater than 2 could be attractive in the sense that it could allow us to obtain the same error level with less sub-grids [13]. In [12,13], it had been shown that for a refinement ratio of 4 , the mesh convergence remains in $O\left(d_{h_{\text {fine }}}\right)$. That is why we decide to compare our LDC method applied on an initial mesh of size $h_{i} / 2$, with 1 to 7 sub-grids of refinement ratio 2 to 1 to 4 sub-grids of refinement ratio 4 . These two approaches are put to the test of a comparison with a global h-refinement method. This method [28] consists in building a unique locally refined conforming mesh on which the simulations are performed. For all approaches, the refined meshes are obtained thanks to the $\mathrm{ZZ}$ a posteriori error estimator, see Fig. 12 for example. The relative errors versus CPU time are reported on Fig. 13. All methods are based on a standard $Q_{1}$ finite element solver.

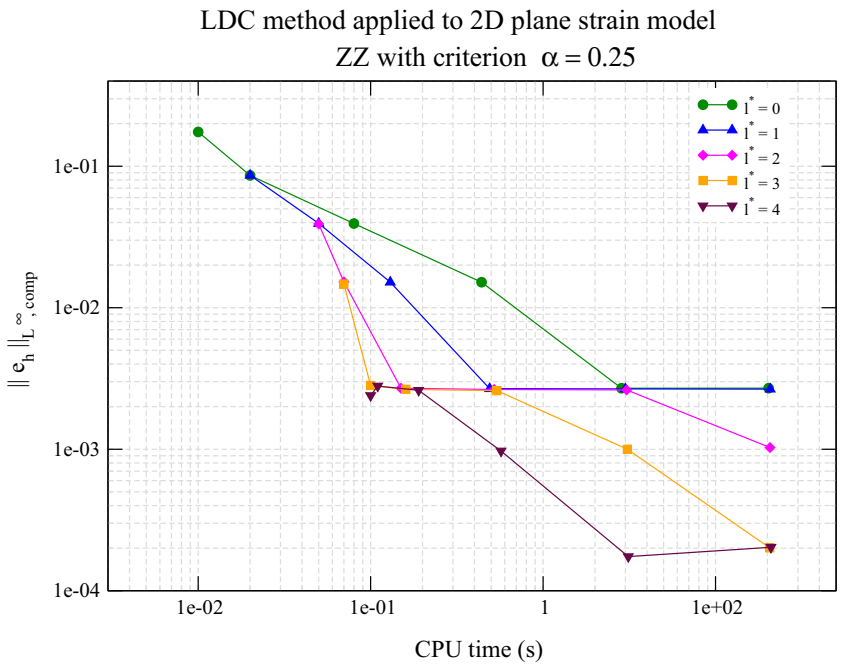

Fig. 11. $L^{\infty}$ error on the composite grid according to CPU time - 2D plane strain model.
Table 1

Total and additional $(+)$ number of nodes $-2 \mathrm{D}(\mathrm{r}, \theta)$ test case.

\begin{tabular}{|c|c|c|c|c|c|}
\hline \multicolumn{6}{|c|}{ Number of nodes according to the meshes } \\
\hline & 0 & 1 & 2 & 3 & 4 \\
\hline$h_{i}$ & 66 & $\begin{array}{l}297 \\
(+231)\end{array}$ & $\begin{array}{l}418 \\
(+121)\end{array}$ & $\begin{array}{l}539 \\
(+121)\end{array}$ & $\begin{array}{l}660 \\
(+121)\end{array}$ \\
\hline$h_{i} / 2$ & 231 & $\begin{array}{l}352 \\
(+121)\end{array}$ & $\begin{array}{l}473 \\
(+121)\end{array}$ & $\begin{array}{l}\mathbf{5 9 4} \\
(+121)\end{array}$ & $\begin{array}{l}715 \\
(+121)\end{array}$ \\
\hline$h_{i} / 4$ & 861 & $\begin{array}{l}982 \\
(+121)\end{array}$ & $\begin{array}{l}1103 \\
(+121)\end{array}$ & $\begin{array}{l}1224 \\
(+121)\end{array}$ & $\begin{array}{l}1345 \\
(+121)\end{array}$ \\
\hline$h_{i} / 8$ & 3321 & $\begin{array}{l}\mathbf{3 4 4 2} \\
(+121)\end{array}$ & $\begin{array}{l}3563 \\
(+121)\end{array}$ & $\begin{array}{l}3684 \\
(+121)\end{array}$ & $\begin{array}{l}3871 \\
(+187)\end{array}$ \\
\hline$h_{i} / 16$ & 13,041 & $\begin{array}{l}13,162 \\
(+121)\end{array}$ & $\begin{array}{l}13,283 \\
(+121)\end{array}$ & $\begin{array}{l}13,470 \\
(+187)\end{array}$ & $\begin{array}{l}13,679 \\
(+209)\end{array}$ \\
\hline$h_{i} / 32$ & 51,681 & $\begin{array}{l}51,802 \\
(+121)\end{array}$ & $\begin{array}{l}51,989 \\
(+187)\end{array}$ & $\begin{array}{l}52,198 \\
(+209)\end{array}$ & $\begin{array}{l}52,385 \\
(+187)\end{array}$ \\
\hline
\end{tabular}

For rough relative errors (about $1 \cdot 10^{-2}$ ) and then small CPU times (about $5 \cdot 10^{-2} \mathrm{~s}$ ) LDC performances for ratios 2 and 4 are comparable to h-refinement strategy ones, even if it seems already attractive in terms of number of nodes. The stagnation observed around a relative error of $3 \cdot 10^{-3}$ is due again to the fact that dividing the local mesh size does not decrease the local distance to the discontinuity. For this special situation, we can then obtain the same error with different number of nodes and different CPU times.

For relative errors less than $3 \cdot 10^{-3}$, the LDC solver becomes attractive, especially with a refinement ratio of 2 . For an error about $3 \cdot 10^{-4}$, the CPU time is for example respectively 20 and 13 times smaller for the LDC method with refinement ratios of 2 and 4 than for the global h-adaptive method.

From a memory space saving point of view, the LDC method is also very attractive. Indeed, even if extra informations are stored (boundary conditions on the levels, factorization of rigidity matrices, right-hand members,...), each local grid is much smaller than an equivalent global refined grid. In particular the total number of nodes of all the sub-grids is far smaller than the number of nodes of the locally adapted grid (we save between $50 \%$ and $92 \%$ of total number of nodes by using LDC with a refinement ratio of 2 , and up to $90 \%$ with a refinement of ratio 4 ).

For small errors, the use of a refinement ratio of 4 instead of 2 is not so attractive. Nevertheless, this conclusion is strongly related to the size of the zone of interest. Indeed, the use of a larger ratio allows to limit the number of sub-grids, but may imply more extended grids and thus more nodes than necessary in some zones for very localised discontinuities (see Fig. 12). For example in our case, there are between $20 \%$ and $35 \%$ less elements for the same error if a refinement ratio of 2 is used instead of 4 . For a linear behaviour, the gain in the number of sub-grids is not advantageous in terms of time consuming since only the first prolongation step is costly (linear behaviour exactly solved). These results remain true as long as no local resolution of the behaviour law is required (see Section 5).

To conclude, the LDC solver seems very attractive. Indeed, for a given error we obtain smaller CPU time and much less elements than using a locally refined mesh.

\subsection{Three-dimensional phenomena}

\subsubsection{Problem definition}

This model gathers both two-dimensional phenomena on a three-dimensional geometry. For symmetry reasons, only $1 / 32$ of the cladding in front of a pellet is represented $\left(R_{\text {int }} \leqslant r \leqslant R_{\text {ext }}, 0\right.$ $\leqslant \theta \leqslant \pi / 8,0 \leqslant z \leqslant L / 2$ ), see Fig. 14. Boundary conditions are those of the $2 \mathrm{D}(r, \theta)$ problem (see Section 4.1.1 and the associated 

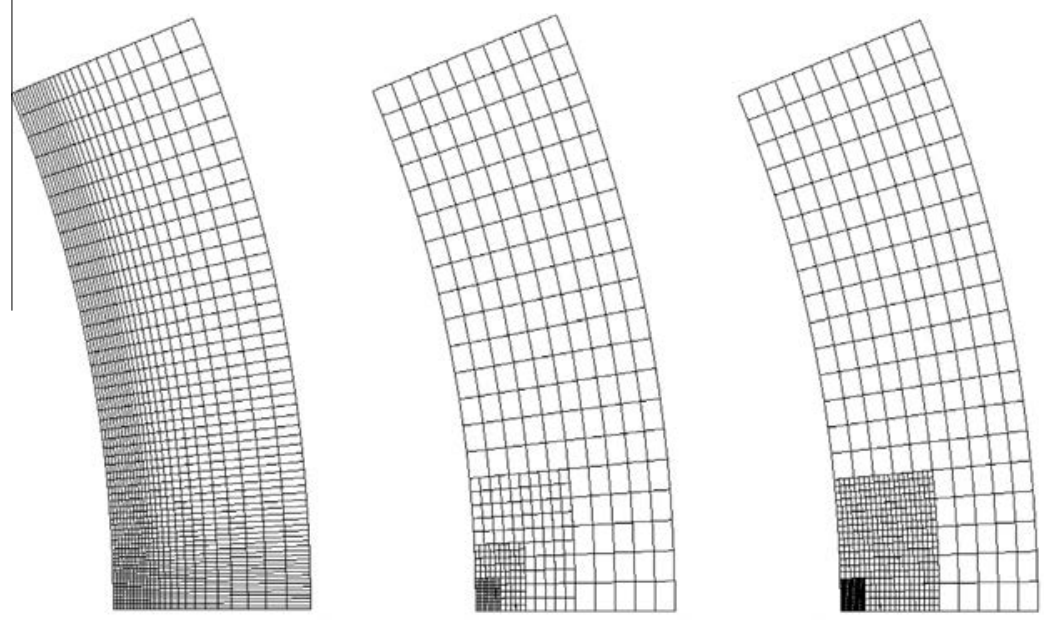

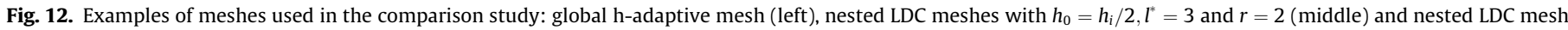
with $h_{0}=h_{i} / 2, l^{*}=2$ and $r=4$ (right)

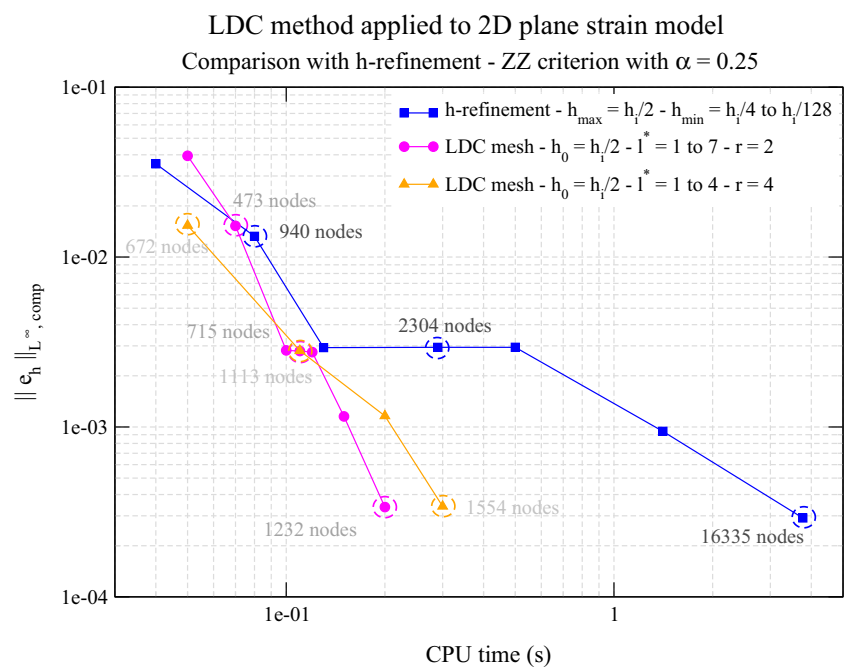

Fig. 13. $L_{\text {comp }}^{\infty}$ error according to CPU time and mesh size - 2D plane strain model Comparison between LDC solver and global h-adaptive method.

notations) completed by those in the axial direction with $L=13.5 \mathrm{~mm}$ and $L_{h}=0.6 \mathrm{~mm}$ :

- Symmetry conditions due to the partial representation of the cladding:

$\mathbf{u} \cdot \mathbf{n}=\mathbf{0}$ on the surfaces $\theta=0, \quad \theta=\pi / 8$ and $z=0$

- Uniform normal displacement on the upper surface:

$\mathbf{u} \cdot \mathbf{n}=$ constant on $z=L / 2, \quad \forall \theta$ and $\forall r$

- Internal discontinuous pressure representing the contact with the cracked pellet:

On $r=R_{\text {int }}$ :

$\sigma \mathbf{n}=0$ Pa on $0 \leqslant \theta \leqslant \theta_{f}, \quad \forall z$

$\sigma \mathbf{n}=-150 \cdot 10^{6} \mathrm{~Pa}$ on $\theta_{f}<\theta \leqslant \pi / 8$ and $z \leqslant L_{h}$

$\sigma \mathbf{n}=-80 \cdot 10^{6}$ Pa on $\theta_{f}<\theta \leqslant \pi / 8$ and $z>L_{h}$

- External pressure imposed by the coolant:

$\sigma \mathbf{n}=-15.5 \cdot 10^{6} \mathrm{~Pa} \quad$ on $r=R_{\text {ext }}, \quad \forall \theta, \forall z$

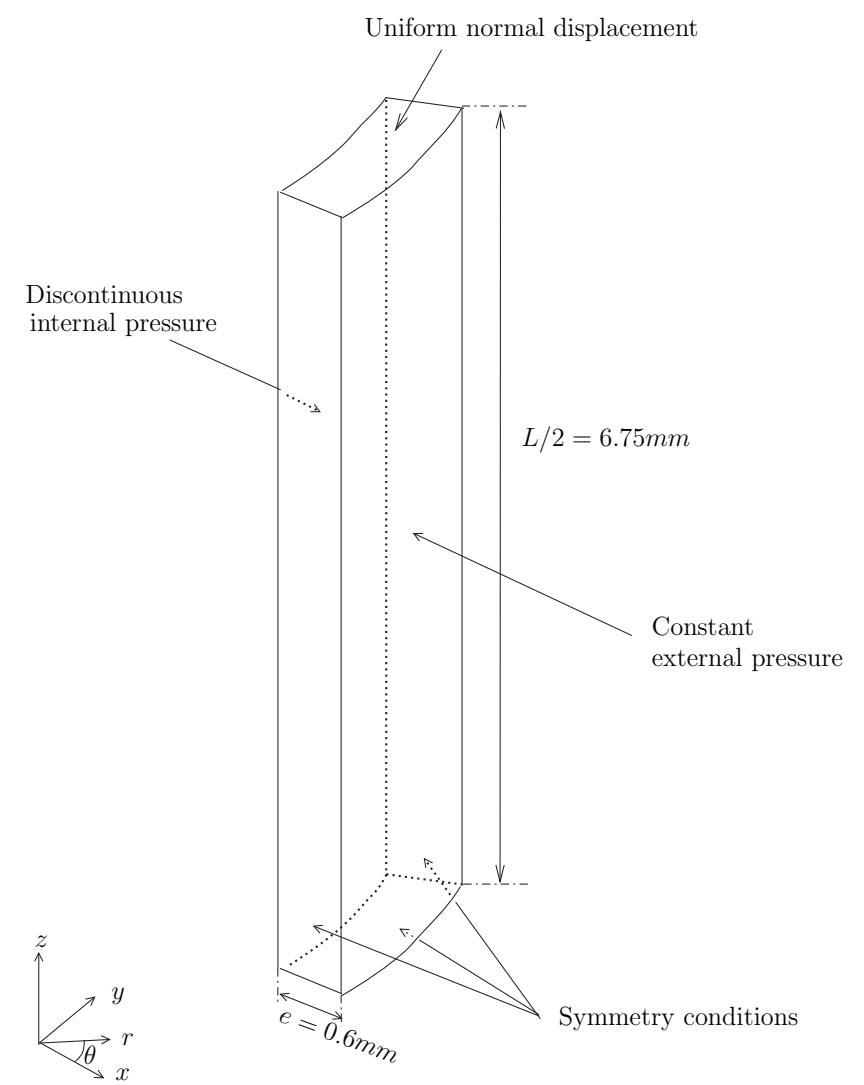

Fig. 14. 3D geometry and problem definition.

The goals are multiple: verify the LDC method performances and verify automatic detection and treatment of several discontinuities of different characteristic length-scale.

We have to notice that, due to the size of the problem under study, we cannot obtain an accurate reference solution with a quasi uniform mesh any more. The reference solution is hence obtained with a mesh of space-step varying from 2 to $50 \mu \mathrm{m}(\simeq 2$ millions of DoF). 


\subsubsection{Mesh convergence study}

We use the same methodology as in section 4.1 and [13] to obtain nested meshes. The refinement parameter $\alpha$ is still set to 0.25 . The coarsest mesh step $h_{i}$ is equal here to $629 \mu \mathrm{m}$. In this case $d_{h}$ represents the distance between the approximated location on the mesh of step $h$ and the real location of the so-called "triple point", which is defined by the intersection of the two pressure discontinuities curves. In view of the conclusions of the linear elastic two-dimensional study, we choose to only use a refinement ratio of 2 for all the linear elastic three-dimensional study.

The meshes obtained thanks to the a posteriori automatic detection are very localised around the two discontinuities, as it can be seen on Fig. 15.

The maximal composite error norm is plotted on Fig. 16 according the distance $d_{h_{0}}$ of the coarse mesh to be refined.

The expected first-order convergence is reached. Moreover, the error improvement due to the refinement strategy remains true in a three-dimensional context, as the method still converges as $O\left(d_{h_{\text {fine }}}\right)$. This result verifies the use of the LDC method coupled with $\mathrm{ZZ}$ a posteriori error estimator to treat three-dimensional problems with crossed discontinuities.

\subsubsection{Interest of regular meshes}

In an engineering context and according to the stretch of the structure, it seems attractive to use stretched meshes in the axial direction. For example the initial mesh on Fig. 17 is two times longer in the height than the previous one. If we set $\alpha=0.25$, the $\mathrm{ZZ}$ a posteriori error estimator does not detect entirely the two discontinuities (see meshes on Fig. 17).

The mesh convergence results obtained with these meshes are far from the expected ones, as it can be seen on Fig. 18 which represents the maximal composite error norm according to the distance $d_{h_{0}}$ to the intersection point on the coarse mesh.

The $O\left(d_{h_{\text {fine }}}\right)$ convergence is no more reached especially for very coarse initial meshes. Moreover, the use of additional sub-grids $\left(l^{*}>1\right)$ does not decrease the obtained error. In this case, the
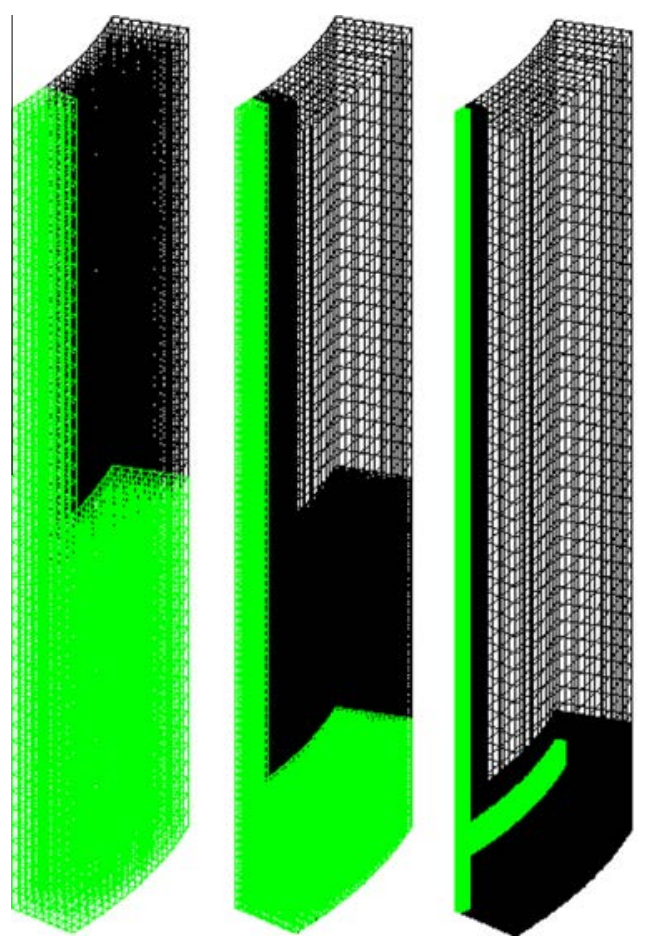

Fig. 15. Example of nested meshes obtained with regular meshes and $\alpha=0.25-3 D$ model.

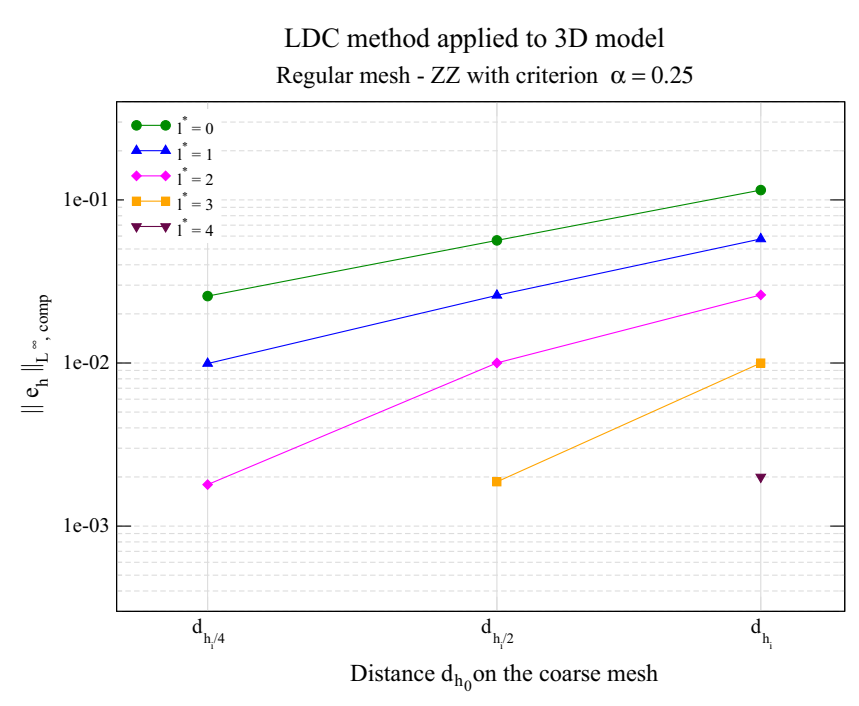

Fig. 16. Composite maximal error norm according to the distance to the triple point $-\alpha=0.25$ - Regular structured meshes - 3D model.
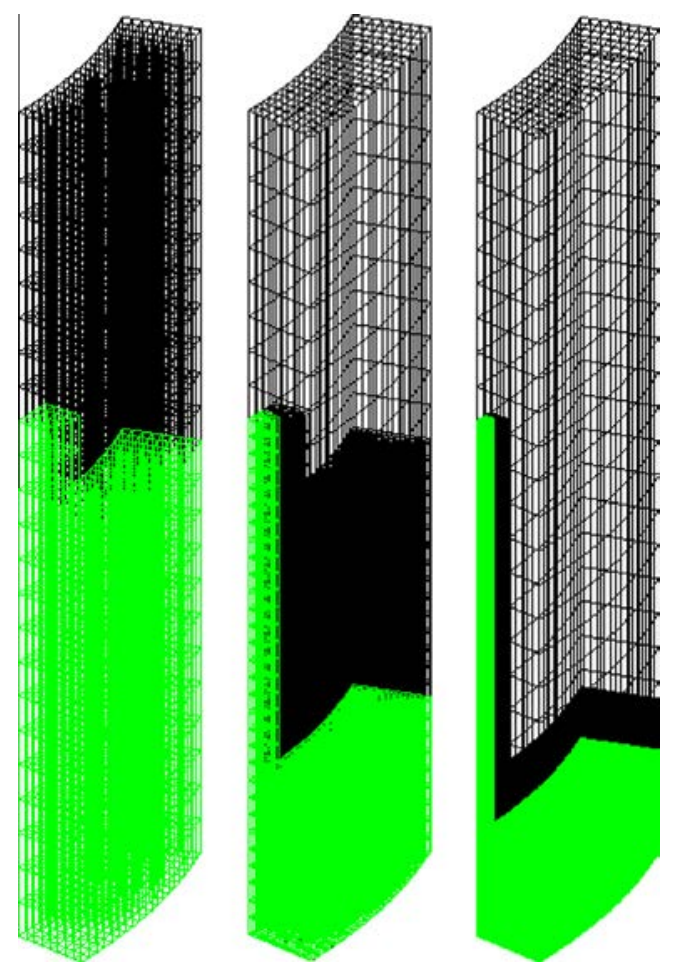

Fig. 17. Example of nested meshes obtained with stretched meshes and $\alpha=0.25$ 3D model.

non-regularity of the mesh around the main discontinuity hides the lower order discontinuity (in the plane $(r, \theta)$ ) from the $\mathrm{ZZ}$ a posteriori error estimator point of view.

A sensibility study shown that the refinement criterion $\alpha$ has to be set to 0.15 to select accurately the two discontinuities. The stretch of the mesh induces a wide detected refinement zone around the hourglass pressure jump (see Fig. 19 compared to Fig. 15). The $\mathrm{ZZ}$ a posteriori error estimator seems less efficient to detect several discontinuities of different characteristic lengthscale if the considered meshes are stretched and coarse. 


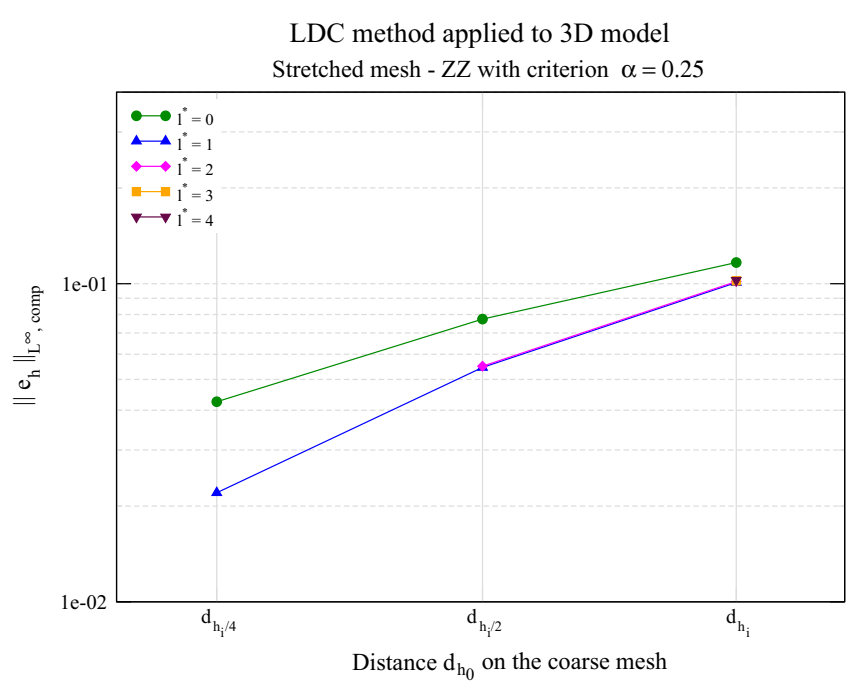

Fig. 18. Composite maximal error norm according to the initial distance to the intersection point $-\alpha=0.25$ with stretched mesh $-3 \mathrm{D}$ model.

The $L^{\infty}$ relative error between the reference solution and the composite LDC solution for $\alpha=0.15$ and stretched meshes is plotted on Fig. 20.

Yet, the expected $O\left(d_{h_{\text {fine }}}\right)$ convergence is reached. However, smaller errors than $10^{-2}$ as obtained on Fig. 16 are not reachable here. Indeed, the number of DoF implied avoid the inversion of the linear system.

Additionally to the well-known performances of linear solvers on structured grids, this study enables us to conclude that the quality of the $\mathrm{ZZ}$ a posteriori error estimator also strongly depends on the regularity of the mesh.

\subsubsection{Comparison with a global h-adaptive refinement technique}

In this part, we use the regular meshes obtained on Fig. 15 with $\alpha=0.25$. A comparison between the LDC method and a global
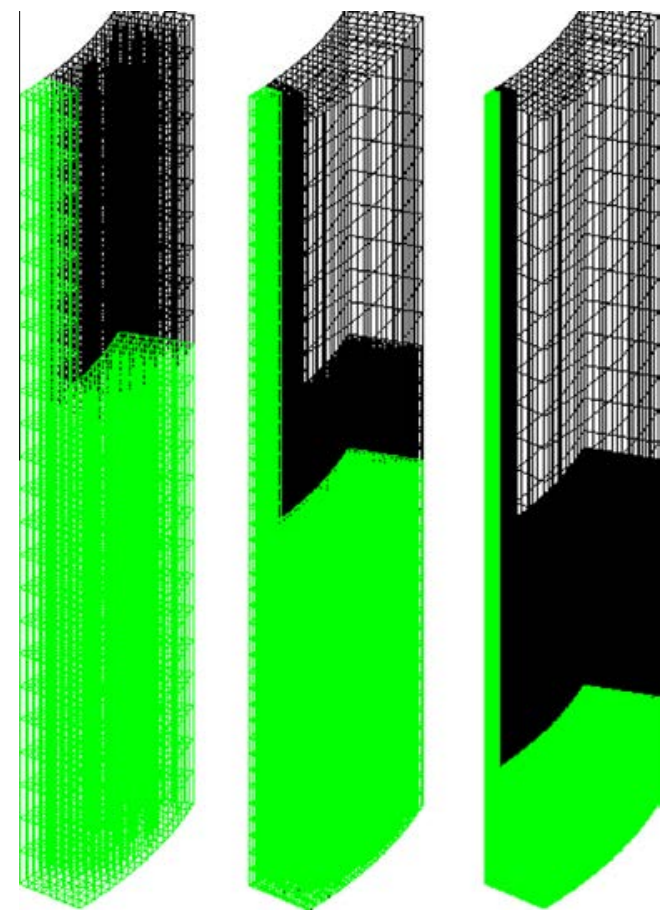

Fig. 19. Example of nested meshes obtained with stretched meshes and $\alpha=0.15$ 3D model.

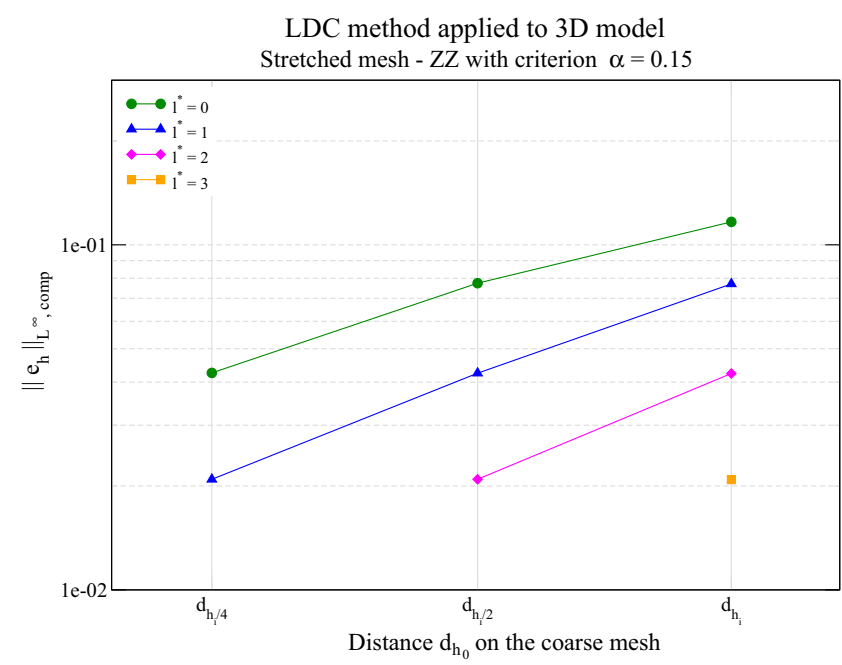

Fig. 20. Composite maximal error norm according to the mesh $-\alpha=0.15-3 D$ model.

$\mathrm{h}$-adaptive resolution is made for the three-dimensional case. Here again, the optimal h-refined mesh is obtained through the ZZ estimation. The results are presented on Fig. 21.

These results are really satisfying. Indeed, for relative errors about $2 \cdot 10^{-2}$, the LDC method and the global h-adaptive method lead to quite equivalent results in terms of CPU time as well as in terms of number of nodes.

For errors smaller than $1 \cdot 10^{-2}$, the LDC solver becomes more and more attractive in terms of memory space and CPU time.

Moreover, the increasing difference in number of nodes between the two methods induces that the global h-refinement method cannot be performed any more for expected errors as precise as those obtained with the LDC solver.

\section{Nonlinear study: Norton creep behaviour}

\subsection{Problem definition}

As the LDC process for solid mechanics has been verified for linear elastic behaviour in previous section, we will now complex the cladding behaviour. A Norton creep behaviour is now studied. It adds to the linear strain $\varepsilon^{e}$ a nonlinear strain $\varepsilon^{v p}$ defined as:

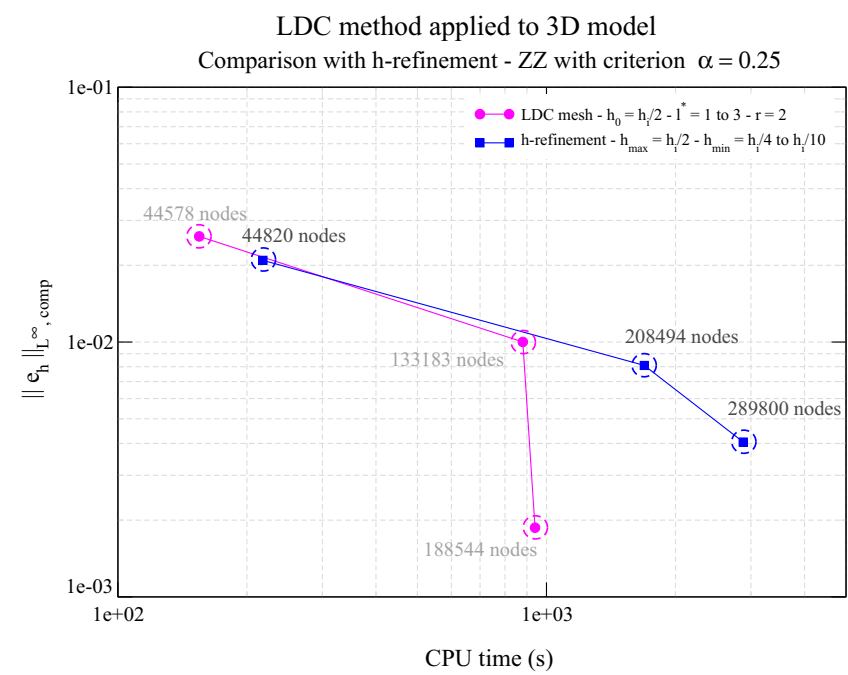

Fig. 21. Comparison between LDC and global h-adaptive resolution - 3D model. 

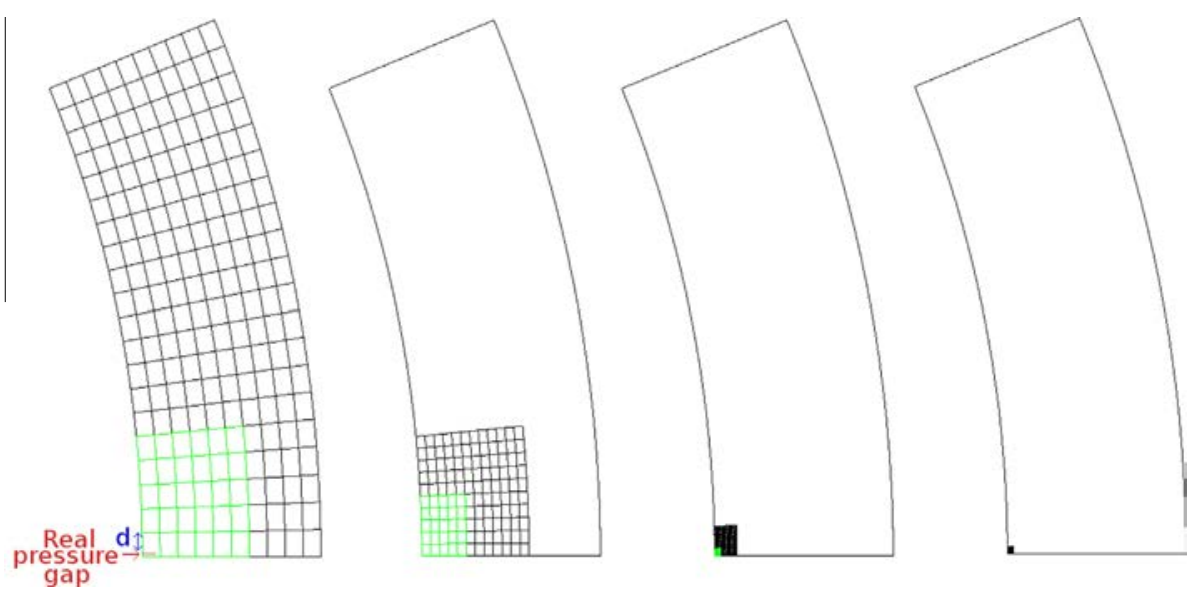

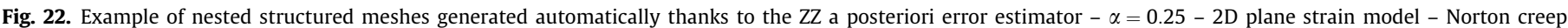
behaviour.

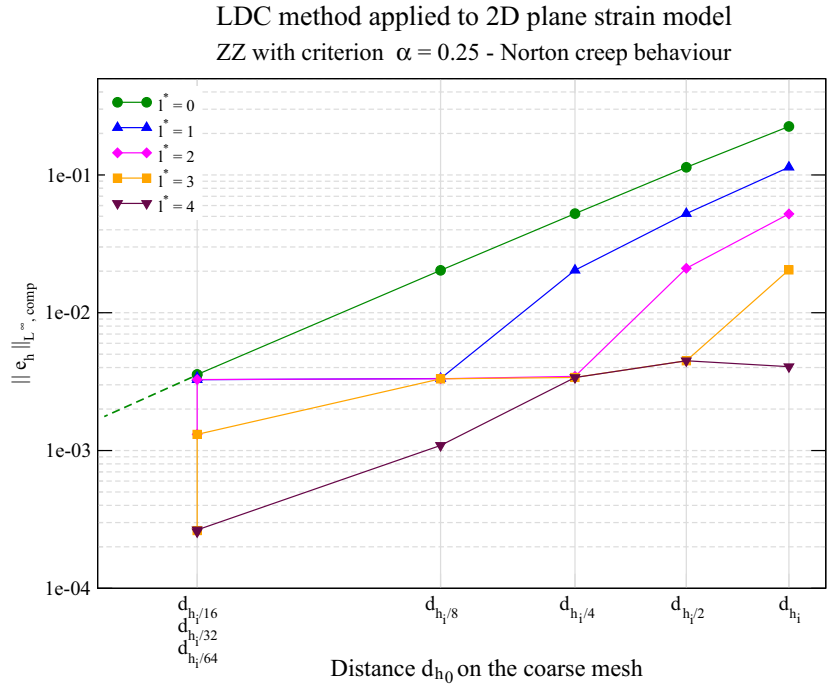

Fig. 23. $L_{\text {comp }}^{\infty}$ error according to the mesh - 2D plane strain model - Norton creep behaviour.

$\dot{\varepsilon}^{v p}=\left(\frac{J}{K}\right)^{n-1} \sigma_{d}$

where $n=5$ and $K=2.6 \cdot 10^{11} \mathrm{~Pa}^{5 / 4} \mathrm{~s}^{1 / 4}$ are two given material coefficients, $\sigma_{d}$ represents the deviatoric stress tensor and $J$ is the second invariant of $\sigma_{d}$.

Young's modulus and Poisson's ratio are those given in Section 3.2: $E=100 \mathrm{GPa}$ and $v=0.3$.

The time-dependent nonlinear problem is solved up through an incremental process applied between the times $t$ and $t+\Delta t$. Since the behaviour law is integrated on each Gauss point, the increment of the solution $\Delta u$ is calculated thanks to a fixed point method based on residual increments [29]. As a first simplified problem, we only consider one time increment of $1 \mathrm{~s}$. The results used in the next figures are the ones obtained after this first increment.

From the LDC algorithm point of view, the main difficulty in this case is linked to the evaluation of the defect. Indeed, this defect implies a restricted stress, which cannot be easily obtained from the restricted displacements as for linear behaviours. In the other hand, reliable projection methods of the stresses (or any other field defined on the Gauss points) do not exist. That is why we chose to still restrict the displacements and then solve the coarse nonlinear problem with this restricted displacement imposed as Dirichlet boundary conditions. The resulting internal forces associated to the calculated stresses then correspond to the coarse operator applied on the restricted solution. Moreover in this nonlinear context, a quasi-exact resolution is really costly. The fine defect is then no longer negligible, and the second term of Eq. (4) has to be taken into account. An interpolation operator which respects the conservation of total forces is then used.

\subsection{Mesh convergence study}

The test case under study is the same two-dimensional problem as the one presented in Section 4.1.1. This study is a first step in applying our automatic process to nonlinear behaviours. As a consequence, we first neglect the time dependence of the mesh generation. In this section, the problem under study is then examined during one time step.

The same methodology as for the linear study is applied to obtain the refinement zones. The coarsest mesh step is $h_{i}=218 \mu \mathrm{m}$. A refinement ratio of 2 is used for the convergence study. On the example of nested meshes on Fig. 22, it must be noticed that the refinement areas (in green) are almost the same than in linear context (see on Fig. 8). It can be explained by the fact that the pressure discontinuity effect is much more influential in terms of mesh refinement than the nonlinear behaviour of the whole structure.

Fig. 23 represents the $L^{\infty}$ relative error $\left\|e_{h}\right\|_{L^{\infty} \text { comp }}$ between the reference displacement solution (obtained with a regular structured mesh of size $4 \mu \mathrm{m}$ that is to say approximatively 0.5 millions DoF) and the nonlinear composite LDC displacement solution.

To the best of our knowledge, there is no theoretical result on the convergence of the LDC method for a nonlinear problem. However, the nonlinear material behaviour does not deteriorate the convergence in $O\left(d_{h_{\text {fine }}}\right)$ of the LDC method obtained for linear behaviour. For some simulations, the error improvement begins to be limited by the pollution error of the non-refined zone [9] (for example $h_{0}=h_{i}$ and $l^{*}=4$ ). As for the linear case, the ratio precision over CPU time or memory space is improved using an initial coarse mesh and a lot of localised sub-grids.

\subsection{Comparison with a global h-adaptive refinement technique}

A comparison between the LDC solver (with refinement ratios of 2 and 4 ) and the standard $Q_{1}$ FE solver applied to global h-adaptive mesh has been also performed for the nonlinear behaviour. 

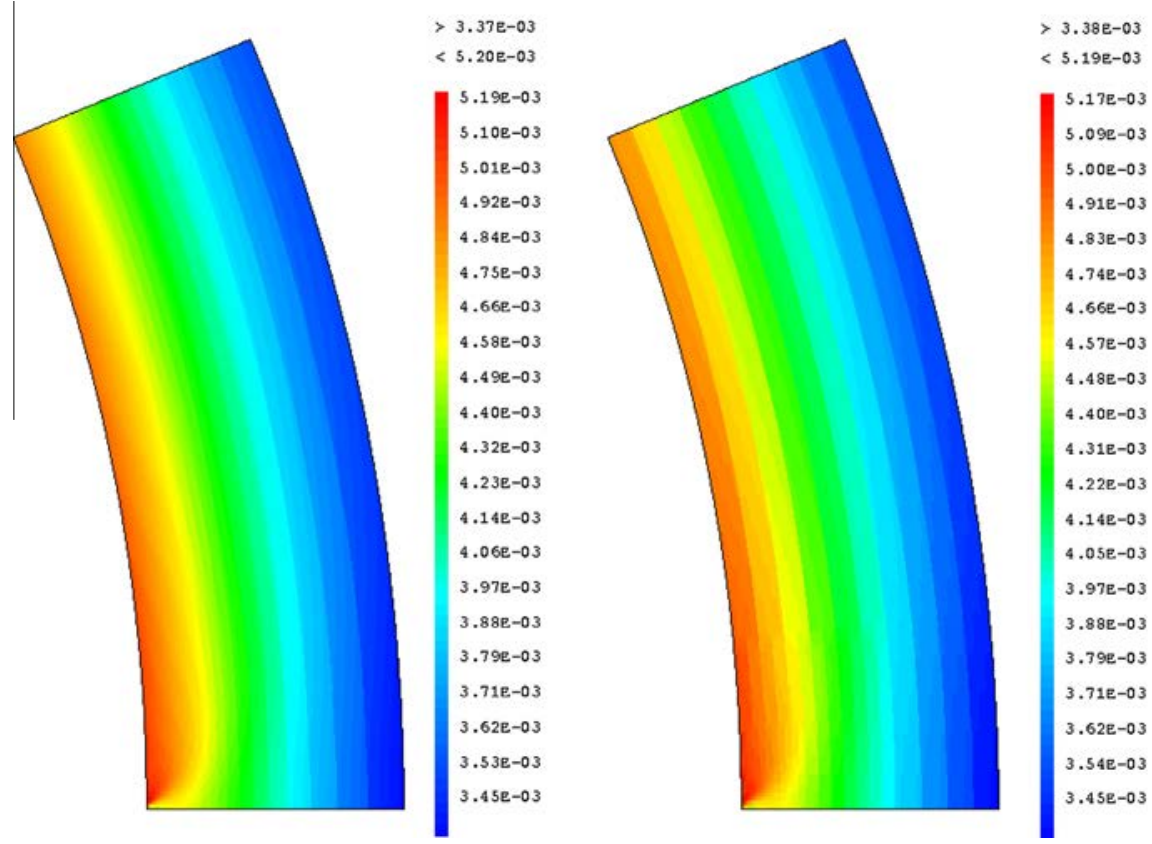

Fig. 24. Equivalent inelastic strain for the global h-adaptive refinement technique (left) and for the composite LDC one with a refinement ratio of 2 (right) at the end of the time increment - 2D plane strain model - Norton creep behaviour.

LDC method applied to 2D plane strain model

Comparison with h-refinement - Norton creep behaviour - ZZ with criterion

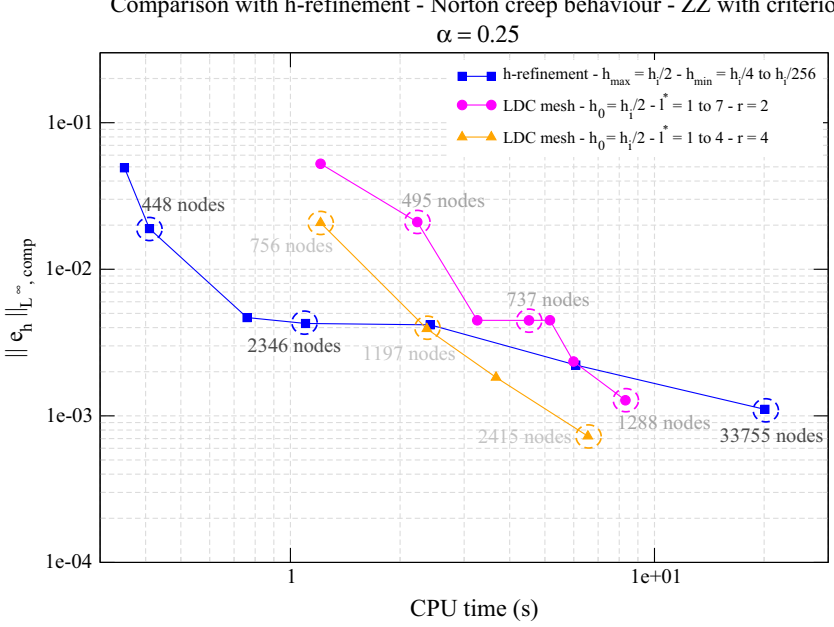

Fig. 25. Comparison between LDC and h-adaptive resolution $-2 D(r, \theta)$ nonlinear model.

The first comparison was made on the equivalent inelastic strain, see Fig. 24. In our test case, the inelastic part of the strain field represents about $60 \%$ of the total strain.

The strain distributions obtained using the h-adaptive resolution and the LDC method are equivalent. Moreover, the maximal relative error is under $0.5 \%$, which is really promising according to the current precision of the physical models.

On Fig. 25, we compare the two refinement strategies, for equivalent local fine and coarse mesh sizes.

First, if we compare the LDC method with refinement ratios of 2 and 4 , we can notice that the use of a refinement ratio of 2 always leads to less nodes than a refinement ratio of 4 . This is due to the strong localisation of the zone of interest. However, a greater refinement ratio induces faster computational times. It can be explained by the fact that, contrary to the linear case, each $\wedge$-cycle is quite as costly as the first one. Indeed, the CPU time required for the integration of the behaviour law stays the same. The use of a refinement ratio of 4 allows to limit the number of sub-grids and most importantly the number of $\wedge$-cycles, that induces a faster resolution.

For relative errors about $2 \cdot 10^{-2}$, the global h-refinement method is attractive. Indeed, with as many nodes as the LDC method with a refinement ratio of 2 , it needs 5 (respectively 3 ) times less CPU time than the LDC with a refinement of 2 (respectively 4). This result can be explained by less integrations of the behaviour law.

The LDC solver becomes attractive for errors smaller than $5 \cdot 10^{-3}$, particularly in terms of number of nodes. Indeed, the global h-refinement produces 3 to 26 times more nodes than the LDC method with a refinement ratio of 2 and 2 to 14 times more nodes than the LDC method with a refinement ratio of 4 . Even if the iterative LDC process implies multiple resolution of each grid, the important saving in number of elements compared to the h-refinement method lies to attractive CPU times for small errors. For example, for errors about $1 \cdot 10^{-3}$, the LDC method with a refinement ratio of 2 allows to save 55\% of CPU time compared with the global h-adaptive strategy, and up to $65 \%$ if a refinement of ratio 4 is used.

This test case gives confidence in the use of the LDC method combined with the Zienkiewicz and Zhu a posteriori error estimator to efficiently simulate time-dependent nonlinear mechanical behaviours.

\section{Conclusion}

The local defect correction (LDC) method, which is an adaptive mesh refinement (AMR) method based on multilevel resolutions, has been applied to a simplified problem stemming from the pellet-cladding interaction in pressurised water reactors. We focused only on the cladding response subject to discontinuous pressures from the fuel pellet.

Firstly, the performances of the LDC method have been verified on a linear behaviour. The local sub-grids are automatically 
generated using the Zienkiewicz and Zhu (ZZ) a posteriori error estimator. The results obtained are very satisfying. The expected theoretical mesh convergence as $O\left(d_{h_{\text {fine }}}\right)$ is obtained, where $d_{h_{\text {fine }}}$ is the distance between the real discontinuity and its approximation on the finest grid which mesh size $h_{\text {fine }}$. Saving of computational time and memory space is thus very large in comparison with a standard resolution based on global h-adaptive meshes. Refinement ratios of 2 and 4 have been tested and compared. For linear studies including localised discontinuities, the use of a refinement ratio of 2 has been shown to be optimal, in terms of CPU time and memory space saving.

The LDC method has also been successfully performed on threedimensional studies implying crossed discontinuities of different characteristic length-scale. In this case, the efficiency of the automatic detection of the areas of interest by the $\mathrm{ZZ}$ a posteriori error estimator has been shown to be strongly related to the regularity of the mesh.

Then, a nonlinear material behaviour has been studied, on one time step. Contrary to the linear elastic case, an additional local equation has to be solved on each Gauss point. The performances obtained for the mesh convergence and the number of nodes remains true. The performances in terms of computational time are also satisfying. Moreover, it has been shown that a refinement ratio greater than 2 can be interesting in terms of CPU time.

The prospects of this study is first to add a temporal dependence of the nonlinear behaviour, with the goal of treating the moving of grids position and size. Different kinds of nonlinear behaviours will then be studied.

Lastly, the contact with the pellet will be modelled. The main difficulty will then lie in the treatment of two grids facing each other in a LDC context.

\section{Acknowledgements}

This work has been achieved in the framework of the PLEIADES project, financially supported by CEA (Commissariat à l'Énergie Atomique et aux Énergies Alternatives), EDF (Électricité de France) and Areva.

\section{References}

[1] Strouboulis T, Haque K. Recent experiences with error estimation and adaptivity - 2. Error estimation for h-adaptive approximations on grids of triangles and quadrilaterals. Comput Methods Appl Mech Eng 1992;100(3):359-430.

[2] Babuska I, Suri M. The optimal convergence rate of the p-version of the finiteelement method. SIAM J Numer Anal 1987;24(4):750-76.

[3] Ghosh S, Manna S. r-Adapted arbitrary Lagrangian-Eulerian finite-element method in metal-forming simulation. J Mater Eng Perform 1993(2):271-82.

[4] Khadra K, Angot P. Caltagirone J, Morel P. Concept de zoom adaptatif en architecture multigrille locale; étude comparative des méthodes L.D.C., F.A.C. et F.I.C.. RAIRO - Modélisation Mathématique et Analyse Numérique 1996;30(1):39-82. in French.
[5] Rachowicz W, Oden J, Demkowicz L. Toward a universal h-p adaptive finiteelement strategy - 3. Design of h-p meshes. Comput Methods Appl Mech Eng 1989;77(1-2):181-212.

[6] Belytschko T, Tabbara M. H-adaptive finite-element methods for dynamic problems, with emphasis on localization. Int J Numer Methods Eng 1993;36(24):4245-65.

[7] Düster A, Rank E. The p-version of the finite element method compared to an adaptive h-version for the deformation theory of plasticity. Comput Methods Appl Mech Eng 2001;190:1925-35.

[8] Babuska I, Strouboulis T, Marthur A, Upadhyay C. Pollution-error in the hversion of the finite-element method and the local quality of a-posteriori error estimators. Finite Elem Anal Des 1994;17:273-321.

[9] Oden J, Feng Y. Local and pollution error estimation for finite element approximations of elliptic boundary value problems. J Comput Appl Math 1996;74:245-93.

[10] Hackbusch W. Local defect correction method and domain decomposition techniques. Computing suppl, vol. 5. Springer-Verlag; 1984. p. 89-113.

[11] Zienkiewicz O, Zhu J. A simple error estimator and adaptive procedure for practical engineering analysis. Int J Numer Methods Eng 1987:24:337-57.

[12] Barbié L, Ramière I, Lebon F. A multilevel technique based on nested local meshes for nonlinear mechanics. In: Topping B, editor. Proceedings of the eighth international conference on engineering computational technology. Stirlingshire, UK: Civil-Comp Press; 2012. http://dx.doi.org/ 10.4203/ccp.100.88. paper 88.

[13] Barbié L, Ramière I, Lebon F. Strategies around the local defect correction multi-level refinement method for three-dimensional linear elastic problems. Comput Struct 2014;30:73-90.

[14] Hackbusch W. Multi-grid methods and applications. Springer series in computational mathematics, vol. 4. Springer-Verlag; 1985.

[15] Brandt A. Multi-level adaptive solutions to boundary-value problems. Math Comput 1977;31:333-90.

[16] Ferket P, Reusken A. Further analysis of the local defect correction method. Computing 1996;56(2):117-39.

[17] Zienkiewicz O, Zhu J. The superconvergent patch recovery and a posteriori error estimation. Part I: The recovery technique. Int J Numer Methods Eng $1992 ; 33: 1331-64$

[18] Zienkiewicz O, Zhu J. The superconvergent patch recovery and a posteriori error estimation. Part II: Error estimates and adaptivity. Int J Numer Methods Eng 1992;33:1365-82.

[19] Babuska I, Rheinboldt W. A-posteriori error estimates for the finite element method. Int J Numer Methods Eng 1978;12:1597-615.

[20] Ladevèze P, Leguillon D. Error estimate procedure in the finite-element method and applications. SIAM J Numer Anal 1983;20:485-509.

[21] Michel B, Sercombe J, Thouvenin G, Chatelet R. 3D fuel cracking modelling in pellet cladding mechanical interaction. Eng Fract Mech 2008;75:3581-98.

[22] CAST3M, <www.cast3m.cea.fr>.

[23] Michel B, Sercombe J, Thouvenin G. A new phenomenological criterion for pellet-cladding interaction rupture. Nucl Eng Des 2008;238:1612-28.

[24] Ramière I. Convergence analysis of the $Q_{1}$-finite element method for elliptic problems with non-boundary-fitted meshes. Int J Numer Methods Eng 2008;75(9):1007-52.

[25] Nonon C, Lansiart S, Struzik C, Plancq D, Martin S, Decroix G, Rambouille O, Beguin S, Julien B. Differential PCI behaviour of PWR fuel rods under transient conditions. In: International topical meeting on LWR fuel performance; 2004.

[26] Roberts $G$. The concentration of stress in cladding produced by the expansion of cracked fuel pellets. Nucl Eng Des 1978;47:257-66.

[27] Ramière I, Angot P, Belliard M. A fictitious domain approach with spread interface for elliptic problems with general boundary conditions. Comput Methods Appl Mech Eng 2007;196:766-81.

[28] Demkowicz L, Devloo P, Oden J. On a h-type mesh-refinement strategy based on minimization of interpolation errors. Comput Methods Appl Mech Eng 1985;53(1):67-89.

[29] Blanc V. Modélisation du comportement thermomécanique des combustibles à particules par une approche multi-échelle [Ph.D. thesis]. Université de Provence, 2009, in French. 